\title{
The Potential Role of Subclinical Bordetella pertussis Infection in Epilepsy
}

\author{
Keith Rubin and Steven Glazer* \\ ILiAD Biotechnologies, Weston, FL, United States
}

Keywords: Bordetella pertussis, epilepsy, seizure, Bordetella pertussis toxin, subclinical infection

The etiology of epilepsy remains unknown in $14-39 \%$ of cases across multiple continents (Banerjee et al., 2009). Given the increased risk for seizures and epilepsy in children after symptomatic Bordetella pertussis (BP) infection (Olsen et al., 2015), an association recognized for nearly a century (Eley, 1930), we briefly review the evidence and propose a role for subclinical BP colonizing infections in epilepsy.

Subclinical BP infections are vastly more prevalent than reported pertussis (Ward et al., 2005). In multiple countries with high $\mathrm{BP}$ vaccination rates, evidence of subclinical $\mathrm{BP}$ infection is demonstrated in $4.8-7.1 \%$ of asymptomatic individuals by nasal swab PCR (Klement et al., 2003; Zhang et al., 2014; Naeini et al., 2015), and in $6.6-14.1 \%$ by serology indicative of infection during the past year (de Melker et al., 2006; De Greeff et al., 2010; Palazzo et al., 2016). Based on serology, investigators in the United States (US) acellular BP vaccine trial estimated the number of undocumented BP infections at 1 to 10 million cases in the US annually from 1997 to 1999 (Ward et al., 2005), years when the CDC reported approximately 7,000 cases per year (http://www.cdc. gov/pertussis/surv-reporting/cases-by-year.html), a ratio of up to 1,400 subclinical BP infections for every reported pertussis case.

Multiple lines of evidence support the hypothesis that subclinical nasopharyngeal BP colonizing infections have unrecognized clinical consequences including epilepsy. B. pertussis secretes pertussis toxin, which compromises the blood-brain barrier in human brain endothelium models (Kugler et al., 2007), as seen in epilepsy (Oby and Janigro, 2006). Murine respiratory BP infection induces inflammatory cytokines in the brain (Loscher et al., 2000), and intracerebroventricular pertussis toxin lowers drug-induced seizure thresholds (Durcan and Morgan, 1991), though findings documented in mice should be interpreted with caution. At the neuronal level, mechanistic plausibility is supported in that pertussis toxin increases excitatory neuronal glutamate release (Cullen et al., 1994) and decreases Gi/o receptor-mediated neuroinhibitory GABA activity (Padgett and Slesinger, 2010), as well as GABA receptor binding (Moss and Vaughan, 1988). In summary, mechanisms by which pertussis infection may play a causal role in epilepsy include immunologic and inflammatory responses to pertussis infection, direct action of pertussis toxin on neurons, and a combination of these factors.

Clinical observation also supports the association between BP and epilepsy. In children $<2$ years of age admitted to the hospital with pertussis, new seizures were reported in $2.3 \%$, and encephalopathy in $0.5 \%$ of patients (Halperin et al., 1999). In BP-associated encephalopathy, elevated antibody titers to BP toxins have been demonstrated with 10-fold higher concentrations in CSF compared with serum, indicating entry of BP antigens to the CNS (Grant et al., 1998). In Denmark between 1978 and 2011, the incidence of epilepsy at 10 years of age was 1.7\% for patients with a history of hospital-diagnosed pertussis, and 0.9\% in a matched cohort [HR 1.7 (95\% CI, 1.32.1)] (Olsen et al., 2015). Almost all of the increased epilepsy risk occurred in the first 1.5 years after clinical pertussis, and did not vary with age at pertussis diagnosis. 
Investigating the hypothesis that subclinical BP colonizing infections are a cause of epilepsy could begin by screening patients presenting with an initial idiopathic seizure. Subjects and controls could be tested for serum BP antibody titers and nasopharyngeal BP by swab PCR. In future BP-seizure risk analyses, BP vaccination status should be accounted for to avoid the confounding effects of vaccine-induced BP immunoglobulins on estimates of BP exposure history. The particular form of BP vaccination is also important since the diphtheria, tetanus toxoid and whole-cell pertussis vaccine (DTP) has been associated with febrile seizures (but not epilepsy) (Barlow et al., 2001), while the combination acellular pertussis vaccine (DTaP) has not (Huang et al., 2010). Of note, some historically reported associations between pertussis vaccination and neurologic disorders may be due to early unmasking of genetically determined disease such as Dravet syndrome in those with sodium channel gene SCN1A mutations (McIntosh et al., 2010). Since these mutations may occur without a prior family history, referral for specialty testing should be considered to help identify all potential causes of new onset seizures.

As subclinical BP colonizing infections are prevalent in highly BP-vaccinated populations, and non-human primate studies demonstrate the failure of DTP and DTaP to prevent

\section{REFERENCES}

Banerjee, P. N., Filippi, D., and Hauser, W. A. (2009). The descriptive epidemiology of epilepsy-a review. Epilepsy Res. 85, 31-45. doi: 10.1016/j.eplepsyres.2009.03.003

Barlow, W. E., Davis, R. L., Glasser, J. W., Rhodes, P. H., Thompson, R. S., Mullooly, J. P., et al. (2001). The risk of seizures after receipt of whole-cell pertussis or measles, mumps, and rubella vaccine. New Engl. J. Med. 345, 656-661. doi: 10.1056/NEJMoa003077

Cullen, G. P., Huston, E., and Dolphin, A. C. (1994). Cycloheximide abolishes pertussis toxin-induced increase in glutamate release from cerebellar granule neurones. Neurosci. Lett. 166, 17-22. doi: 10.1016/0304-3940(94)90830-3

De Greeff, S. C., De Melker, H. E., Van Gageldonk, P. G., Schellekens, J. F., van der Klis, F. R., Mollema, L., et al. (2010). Seroprevalence of pertussis in The Netherlands: evidence for increased circulation of Bordetella pertussis. PLoS ONE 5:e14183. doi: 10.1371/journal.pone.0014183

de Melker, H. E., Versteegh, F. G., Schellekens, J. F., Teunis, P. F., and Kretzschmar, M. (2006). The incidence of Bordetella pertussis infections estimated in the population from a combination of serological surveys. J. Infect. 53, 106-113. doi: 10.1016/j.jinf.2005.10.020

Durcan, M. J., and Morgan, P. F. (1991). Intracerebroventricular pertussis toxin enhances sensitivity to N-methyl-d-aspartate-induced seizures in mice. Eur. J. Pharmacol. 197, 209-211. doi: 10.1016/0014-2999(91)90523-S

Eley, R. C. (1930). Neurological complications of whooping cough. New Engl. J. Med. 203, 162-167. doi: 10.1056/NEJM193007242030405

Grant, C. C., McKay, E. J., Simpson, A., and Buckley, D. (1998). Pertussis encephalopathy with high cerebrospinal fluid antibody titers to pertussis toxin and filamentous hemagglutinin. Pediatrics 102(4 Pt 1), 986-990. doi: $10.1542 /$ peds.102.4.986

Halperin, S. A., Wang, E. E., Law, B., Mills, E., Morris, R., Dery, P., et al. (1999). Epidemiological features of pertussis in hospitalized patients in Canada, 19911997: report of the Immunization Monitoring Program-Active (IMPACT). Clin. Infect. Dis. 28, 1238-1243. doi: 10.1086/514792

Huang, W.-T., Gargiullo, P. M., Broder, K. R., Weintraub, E. S., Iskander, J. K., Klein, N. P., et al. (2010). Lack of association between acellular pertussis vaccine and seizures in early childhood. Pediatrics 126, 263-269. doi: $10.1542 /$ peds.2009-1496 nasopharyngeal BP colonization (Warfel et al., 2014), evidence suggests that current pertussis vaccines do not prevent nasopharyngeal BP colonization. Since the number of subclinical BP infections may be more than 1,000 times greater than clinically reported cases as noted above, it would not be surprising to observe a minimal or even lack of epilepsy risk reduction following DTP and DTaP vaccination.

In light of the available evidence, we suggest that a causal role for subclinical BP colonizing infection in epilepsy is plausible and worthy of further investigation. Regression analysis of epilepsy risk, incorporating BP screening assays, medical history, and pertussis vaccination status would be a compelling first step in assessing the potential relationship between epilepsy and subclinical BP infection.

\section{AUTHOR CONTRIBUTIONS}

KR and SG contributed equally to the preparation of this manuscript.

\section{FUNDING}

This work was supported by ILiAD Biotechnologies.

Klement, E., Uliel, L., Engel, I., Hasin, T., Yavzori, M., Orr, N., et al. (2003). An outbreak of pertussis among young Israeli soldiers. Epidemiol. Infect 131, 1049-1054. doi: 10.1017/S09502688030 01110

Kugler, S., Bocker, K., Heusipp, G., Greune, L., Kim, K. S., and Schmidt, M. A. (2007). Pertussis toxin transiently affects barrier integrity, organelle organization and transmigration of monocytes in a human brain microvascular endothelial cell barrier model. Cell. Microbiol. 9, 619-632. doi: 10.1111/j.1462-5822.2006.00813.x

Loscher, C. E., Donnelly, S., Lynch, M. A., and Mills, K. H. (2000). Induction of inflammatory cytokines in the brain following respiratory infection with Bordetella pertussis. J. Neuroimmunol. 102, 172-181. doi: 10.1016/S0165-5728(99)00177-0

McIntosh, A. M., McMahon, J., Dibbens, L. M., Iona, X., Mulley, J. C., Scheffer, I. E., et al. (2010). Effects of vaccination on onset and outcome of Dravet syndrome: a retrospective study. Lancet Neurol. 9, 592-598. doi: 10.1016/S1474-4422(10)70107-1

Moss, J., and Vaughan, M. (1988). ADP-ribosylation of guanyl nucleotide-binding regulatory proteins by bacterial toxins. Adv. Enzymol. Relat. Areas Mol. Biol. 61, 303-379. doi: 10.1002/9780470123072.ch6

Naeini, A. E., Zaman, N., Khorvash, F., and Naeini, S. E. (2015). Does working in hospital increases seroprevalence and carrier state against Bordetella pertussis? Adv. Biomed. Res. 4:194. doi: 10.4103/2277-9175.1 66155

Oby, E., and Janigro, D. (2006). The blood-brain barrier and epilepsy. Epilepsia 47, 1761-1774. doi: 10.1111/j.1528-1167.2006.00817.x

Olsen, M., Thygesen, S. K., Østergaard, J. R., Nielsen, H., Henderson, V. W., Ehrenstein, V., et al. (2015). Hospital-diagnosed pertussis infection in children and long-term risk of epilepsy. JAMA 314, 1844-1849. doi: 10.1001/jama.2015.13971

Padgett, C. L., and Slesinger, P. A. (2010). GABAB receptor coupling to G-proteins and ion channels. Adv. Pharmacol. 58, 123-147. doi: 10.1016/S1054-3589(10)58006-2

Palazzo, R., Carollo, M., Fedele, G., Rizzo, C., Rota, M. C., Giammanco, A., et al. (2016). Evidence of increased circulation of Bordetella pertussis in the Italian adult population from seroprevalence data (2012-2013). J. Med. Microbiol. 65:649-657. doi: 10.1099/jmm.0.000264 
Ward, J. I., Cherry, J. D., Chang, S. J., Partridge, S., Lee, H., Treanor, J., et al. Edwards, K. (2005). Efficacy of an acellular pertussis vaccine among adolescents and adults. New Engl. J. Med. 353, 1555-1563. doi: 10.1056/NEJMoa050824

Warfel, J. M., Zimmerman, L. I., and Merkel, T. J. (2014). Acellular pertussis vaccines protect against disease but fail to prevent infection and transmission in a nonhuman primate model. Proc. Natl. Acad. Sci. U.S.A. 111, 787-792. doi: $10.1073 /$ pnas. 1314688110

Zhang, Q., Yin, Z., Li, Y., Luo, H., Shao, Z., Gao, Y., et al. (2014). Prevalence of asymptomatic Bordetella pertussis and Bordetella parapertussis infections among school children in China as determined by pooled realtime PCR: a cross-sectional study. Scand. J. Infect. Dis. 46, 280-287. doi: $10.3109 / 00365548.2013 .878034$
Conflict of Interest Statement: KR and SG are employed by and hold an equity interest in ILiAD Biotechnologies, which is developing a vaccine for the prevention of Bordetella pertussis. ILiAD Biotechnologies had no role in the study design, analysis, and development of this opinion submission.

Copyright (๑) 2019 Rubin and Glazer. This is an open-access article distributed under the terms of the Creative Commons Attribution License (CC BY). The use, distribution or reproduction in other forums is permitted, provided the original author(s) and the copyright owner(s) are credited and that the original publication in this journal is cited, in accordance with accepted academic practice. No use, distribution or reproduction is permitted which does not comply with these terms. 